
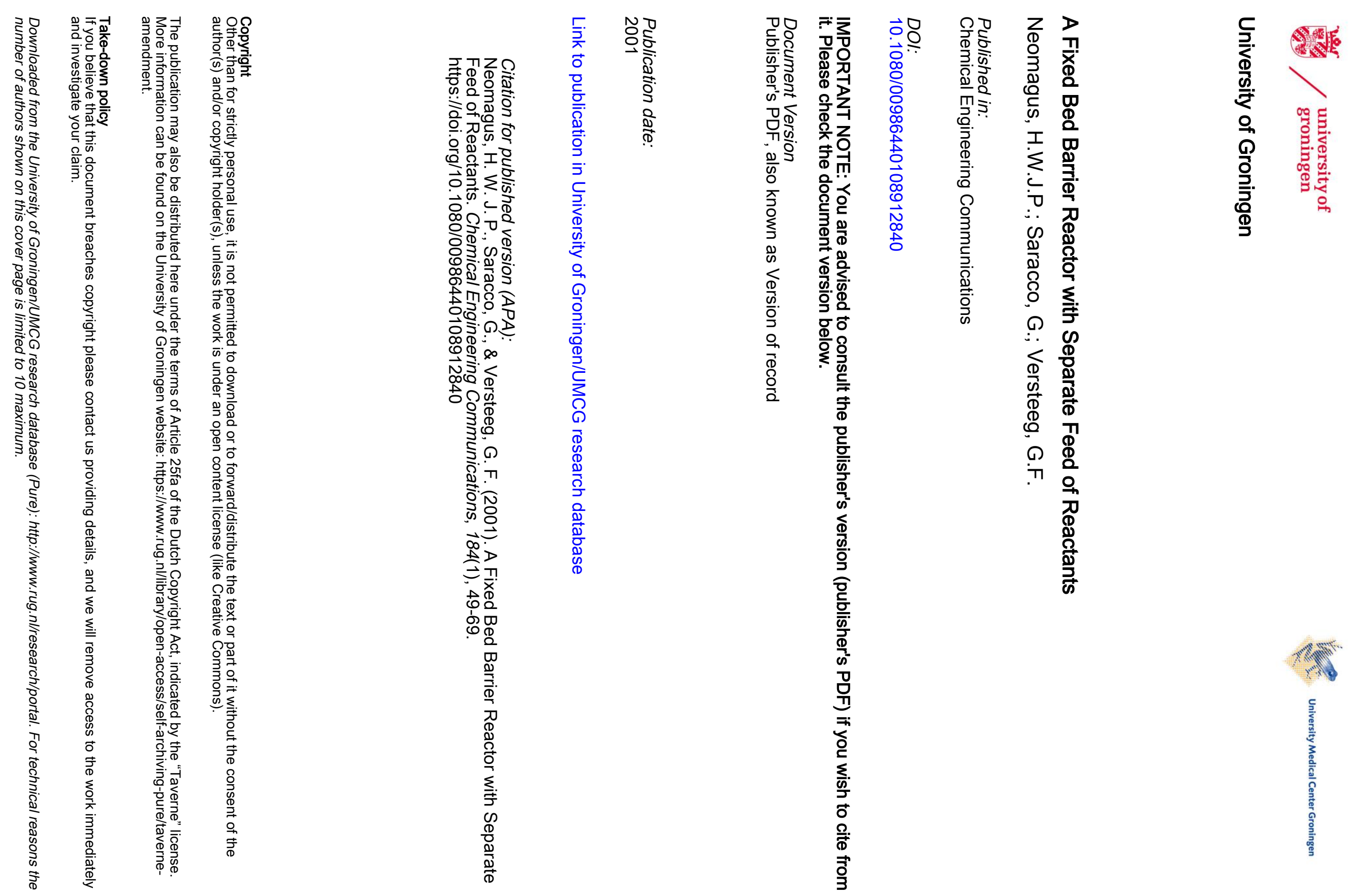
Chem. Eng. Comm., 2001, Vol. 184, pp. 49-69 Reprints available directly from the publisher Photocopying permitted by license only
(1) 2001 OPA (Overscas Publishers Association) N.V. Publisted by license under the Gordon and Breach Science Publishers imprint. Printed in Maluysis.

\title{
A FIXED BED BARRIER REACTOR WITH SEPARATE FEED OF REACTANTS
}

\author{
H. W. J. P. NEOMAGUS ${ }^{a}$, G. SARACCO ${ }^{\mathrm{b}, *}$ \\ and G. F. VERSTEEG ${ }^{\mathrm{a}}$ \\ "Potchefstroom University for CHE, School of Chemical and Minerals Engineering \\ Private Bag X6001, Potchefstroom 2520, Republic of South Africa; ${ }^{b}$ Dipartimento \\ di Scienza dei Materiali e Ingegneria Chimica, Politecnico di Torino, \\ C.so Duca degli Abruzzi 24, I0I29 Torino, Italy
}

(Received 26 October 1999; In final form 22 March 2000)

\begin{abstract}
A new type of gas-solid reactor was developed and characterised in the series of reactor configurations with separate feed of reactants studied by our group. The novelty in the proposed design lies in the use of a fixed bed of small catalytic particles instead of a porous catalytic membrane. The major advantages of this concept, as opposed to the catalytic membrane reactor, are: (i) the barrier activity and pore structure are more homogencous (good properties for modelling purposes); (ii) an easier integration of the catalyst bed into the reactor is obtained (the fixed bed can be sealed more easily into metallic modules than a catalytic membrane).

A Pt catalyst supported on La-stabilised alumina pellets $\left(d_{p}=5-50 \mu \mathrm{m}\right)$ was used and the catalytic oxidation of carbon monoxide was studied as a model reaction to characterise the reactor. A mathematical model based on the Dusty Gas Model approach was conceived and a good agreement between calculations and experiments was obtained. This fixed bed barrier reactor may thus represent a quicker and easier opportunity, compared to membrane reactors, to check the concept of the separate-feed of reactants with new reactions and catalysts.
\end{abstract}

Keywords: Fixed bed barrier reactor; CO oxidation; Pt catalyst; Membrane reactor; Separate reactants feed; Dusty Gas Model

\section{INTRODUCTION}

Although the majority of membrane reactors are using the permselective properties of the membrane to enhance the per-pass conversion of equilibrium limited reactions, Sloot et al. [1] reported of a novel type of

\footnotetext{
*Corresponding author. Tel./Fax: +39-011-5644654/99, e-mail: Saracco@polito.il
} 
catalytic membrane reactor in which membrane permselectivity is not crucial. According to this reactor concept, the membrane acts as a physical barrier, separating two reactants fed at opposite sides of it. This concept has some distinct advantages above other types of gas-solid reactors, in which premixing of the reactants cannot be avoided. Sloot et al. [1] demonstrated that this membrane reactor was particularly attractive for reactions that require a strict stoichiometric feed of reactants (in their study: the catalytic reduction of nitric oxide with ammonia): when reactant concentrations are varied at opposite membrane sides, the reaction zone inside the membrane shifts to reach, once again, a stoichiometric ratio between the incoming reactants fluxes. Conversely, Veldsink et al. [2] showed that this membrane reactor can also be applied for kinetically fast, strongly-exothermic reactions, whose conversion rate becomes mass transfer limited. Thermal problems like explosions and thermal runaways can thus be hindered.

Sloot et al. [1] and Veldsink et al. [2] tested the concept of separate feed of reactants with macroporous $\left(0.1 \mu \mathrm{m}<d_{p}<1 \mu \mathrm{m}\right)$ ceramic membranes. However, the use of this type of membranes is complicated by the catalyst deposition, not easily controllable as concerns homogeneity and activity [3]. Moreover, the implementation of the ceramic membrane in the reactor module is still very difficult and/or expensive: a complex ceramic-to-stainless steel coupling is required. This inconvenience could be only in part reduced by using easily-welded porous-stainless-steel membrane, at the price of a lower high-temperature resistance [4].

In the present study, the membrane is replaced by an ordinary, homogeneous fixed bed of very small catalytic particles $\left(d_{p}=20 \mu \mathrm{m}\right.$ on average), sandwiched between two inert porous filters. The application potential of this fixed-bed barrier reactor (FBBR) will be assessed.

\section{THEORY}

\subsection{Mass Transfer and Chemical Reaction in a FBBR}

Mass transfer in porous systems with a chemical reaction can be described via various approaches. Veldsink et al. [2] showed that whenever, besides diffusion, also a pressure difference affects substantially the mass transfer, the Dusty Gas Model [5] must be used instead of the mathematically simpler Fickian models. The mentioned Authors described the mass flux in the membrane as a combination of bulk- and Knudsen diffusion and the convective contribution was directly implemented in the flux equations using the Darcy equation. 
In this paper, mass transfer with chemical reaction in the fixed bed barrier will be described in a similar way. It must be noted however, that this approach was only experimentally tested for ceramic $[1,2,6]$ and sintered metal membranes [4], so far. In the present reactor the barrier actually consists of three zones, namely the fixed bed and the two filters between which the catalytic layer is sandwiched (Fig. I). The two filters are assumed to be inert: all the catalytic conversion takes place in the fixed bed.

The basic equations describing the mass transfer combined with reaction in an isothermal FBBR reactor are listed in Figure 1.

The structure of the porous medium (the fixed bed and the filters) is characterised by three effective parameters, $K_{0}, B_{0}$ and $\varepsilon / \tau$ (see equations at point $d$ in Fig. 1). As described in [6], $K_{0}$ and $B_{0}$ can be estimated by permeation of a pure gas, whereas $\varepsilon / \tau$ can be determined by an estimation of the effective diffusion coefficients of the reactants in the fixed bed (see later Section 2.3.1). Once such parameters are experimentally determined and the other constitutive parameters evaluated through suitable relationships

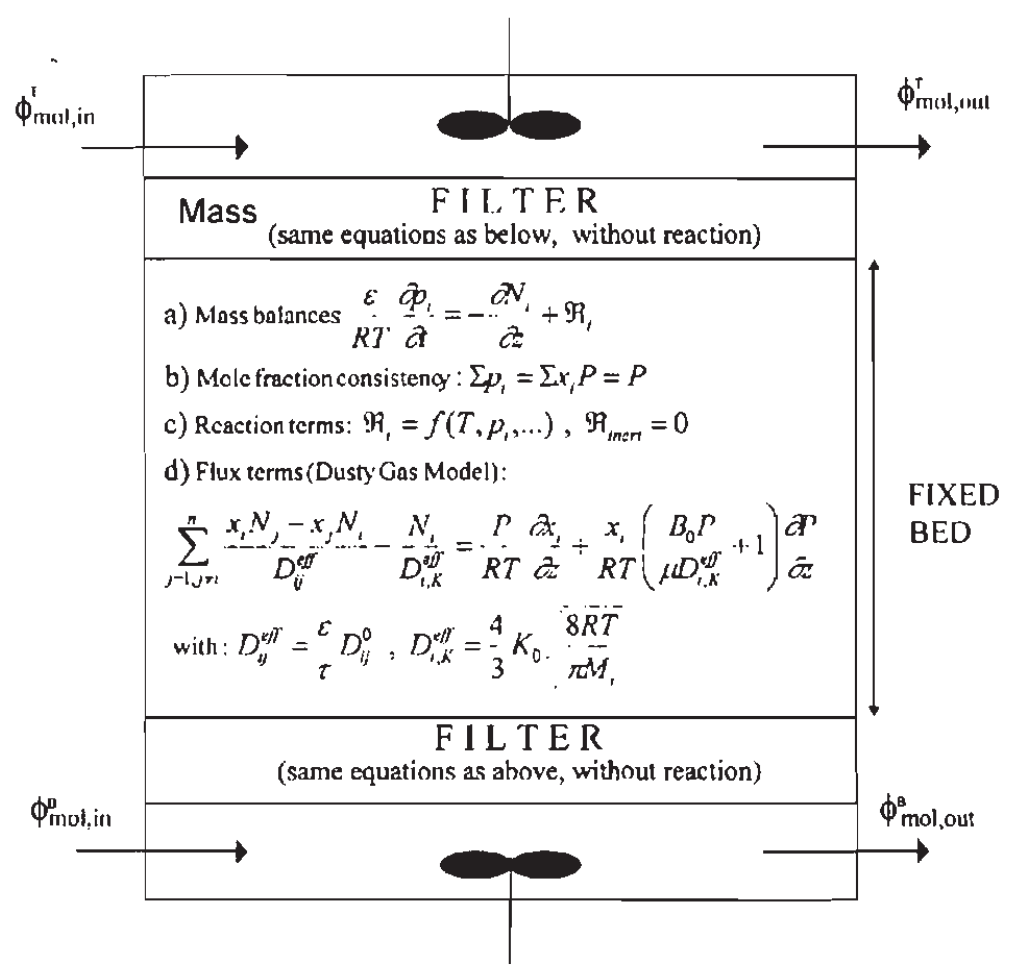

FIGURE 1 Model equations for a fixed-bed barrier reactor with separate feed of reactants. 
(Fig. 2), on the grounds of proper boundary conditions and initial values (see next paragraph) the overall reactant conversion and trans-barrier concentration and pressure profiles can be evaluated by the model. Since all the experimental data here described will be derived under operating conditions almost completely controlled by transport phenomena, reactions kinetics will be considered as virtually infinitely fast. This allows one to neglect reaction rate terms $\boldsymbol{R}$ in the mass balance equations and to relate the fluxes of reactants to the stoichiometric coefficients only. The model was solved through a $a d-h o c$ programme compiled in Pascal (Delphi 3 platform) and running on a PC.

\subsection{Boundary Conditions}

The proposed model for the barrier reactor can only be solved when proper boundary conditions are incorporated. Different types of boundary conditions can be applied as summarised in [6]. The thickness of the barrier in this study is approximately one order of magnitude larger than the conventional ceramic and stainless steel membranes studied in our previous investigations (see Tab. l). Further, the external mass transfer was already demonstrated to affect only marginally the total mass transfer rate (e.g. $[2,4])$. Therefore, it seemed justified to incorporate simple Dirichlet-type boundary conditions: the component concentrations and the pressure at the outer sides of the filters are taken equal to those present in the bulk of the chambers separated by the barrier, the same holding for the filter-fixed bed interfaces. Such chambers are assumed to be perfectly mixed, which allows us to set the above bulk concentration values equal to the concentrations measured experimentally at the exit of each chamber.

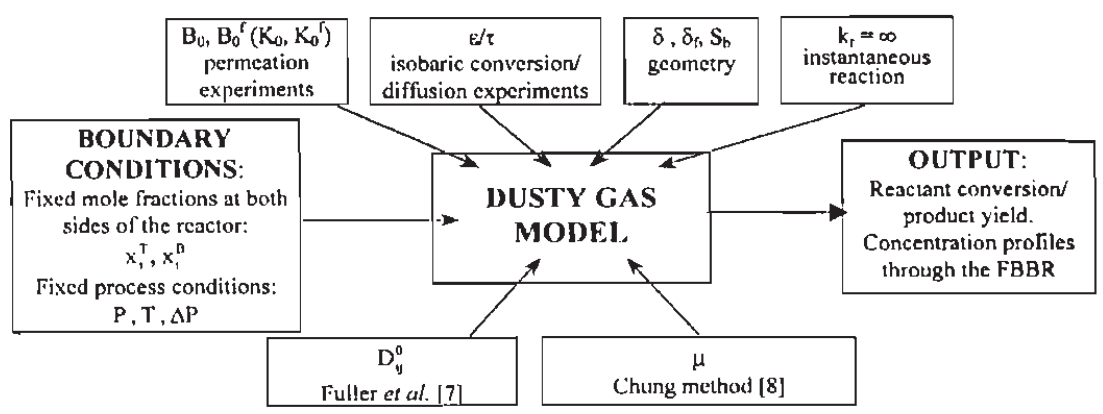

FIGURE 2 Relevant input parameters for the presented model. 
TABLE I Membranes used in previous studies for the concept of separate feed of reactants

\begin{tabular}{lcccc}
\hline Reference & {$[1]$} & {$[2,6]$} & {$[4]$} & This study \\
\hline Material & $\alpha$-Alumina & $\alpha$-Alumina & sintered metal & catalyst powder \\
Geometry & disc & tube & tube & disc \\
$B_{0}\left(\mathrm{~m}^{2}\right)$ & $2.8 \cdot 10^{-16}$ & $7.8-12.3 \cdot 10^{-15}$ & $7 \cdot 10^{-14}$ & $2.1 \cdot 10^{-13}$ \\
$K_{0}(\mathrm{~m})$ & $1.1 \cdot 10^{-8}$ & $10-12.5 \cdot 10^{-9}$ & Negligible effect & $3.49 \cdot 10^{-6}$ \\
$\varepsilon / \tau(-)$ & 0.11 & $0.06-0.07$ & 0.06 & 0.29 \\
$\left\langle r_{p}\right\rangle(\mu \mathrm{m})$ & 0.15 & $0.3-0.4$ & 3 & 3 \\
$\delta(\mathrm{mm})$ & 4.5 & 3 & 2 & 52 \\
\hline
\end{tabular}

\subsection{Characterisation of the Barrier}

In the present section details will be given about the determination of the basic structural and permeation parameters of the fixed bed reactor and of the filters. The series of experiments performed, which have also been successfully used for the sintered-stainless-steel membrane reactor [4], is summarised in Figure 3.

Both the diffusion (Fig. 3a) and isobaric transport-limited conversion (Fig. 3c) measurements provide with quantitative information of the value of $\varepsilon / \tau$, whereas the permeation experiment (Fig. 3b) give exclusively the quantitative value of $B_{0}$ and $K_{0}$. The presence of the filter did not influence significantly the determination of the structural parameters of the fixed bed because the filters are selected in such a way that they have an $\varepsilon / \tau$ ratio of about 0.29 , almost the same as the fixed bed (see later on Sections 4.1 and 4.3), and a negligible pressure drop (i.e., much larger pore size). Equivalent experiments as those outlined in Figures $3 \mathrm{a}$ and $3 \mathrm{~b}$ were in fact also performed on the filters alone to characterise their structure.

When the mentioned structural parameters are determined, final runs in which reaction and pressure difference are combined (Fig. 3d), will be used to verify whether a fixed bed barrier performs similarly to membrane reactors, and whether the model gives accurate predictions of the achievable conversions with no use of fitting parameters.

\subsubsection{Diffusion Experiments}

In the absence of a pressure difference over the porous medium, when no reaction occurs, the flux of a component $i$ can be described according to Fick's law:

$$
N_{i}=\frac{-D_{i}^{e f f}}{R T} \frac{p_{i}^{H}-p_{i}^{L}}{\delta}
$$




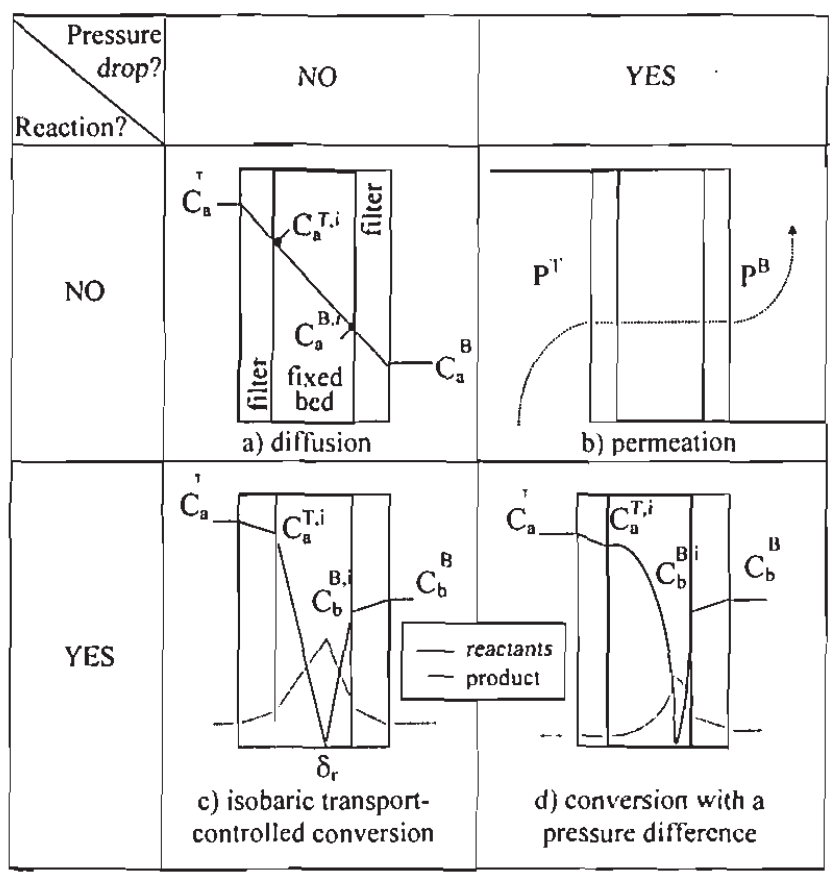

FIGURE 3 Experiments for the structural characterisation of the fixed bed barrier reactor.

Because of the large thickness of the barrier, the external transfer resistance can be neglected (see Section 2.2) and due to the relatively large pore dimensions, the dominant mass-transfer mechanism will be molecular diffusion. The effective diffusion coefficient $D_{i}^{e f f}$ can be directly estimated from the diflusive flux as the driving force is known. The $\varepsilon / \tau$ ratio can now be calculated with:

$$
\frac{\varepsilon}{\tau}=\frac{D_{i}^{e f f}}{D_{i}^{0}}
$$

where the bulk diffusion coefficient $D_{i}^{0}$ is estimated using the method of Fuller [7].

\subsubsection{Permeation Experiments}

For the determination of $B_{0}$ and $K_{0}$, a single gas is forced to permeate the barrier. In this case, the proposed DGM mass-transfer balance will reduce 
to the following, rather straightforward, linear equation (see e.g. [2]):

$$
\frac{\bar{P}}{P} \frac{\phi_{v} \delta}{S_{b}}=\frac{B_{0}}{\mu} \frac{P^{H}+P^{L}}{2}+\frac{4}{3} K_{0} \sqrt{\frac{8 R T}{\pi M}}
$$

$B_{0}$ and $K_{0}$ can thus be estimated directly (from the slope and the intercept of the plots of left-hand-side member $v s$. the average membrane pressure, $\bar{P}$ ) by measuring the pressure difference over the membrane, $\Delta P$, as a result of a given gas flow permeating the barrier at various $\bar{P}$ values.

As it was reported by Neomagus et al. [4], the influence of Knudsen diffusion on the total mass transfer rate when the barrier consists of relatively large pores $(>1 \mu \mathrm{m})$ is almost negligible, and the quantitative value of $K_{0}$ is therefore on the one hand difficult to estimate accurately, but on the other hand not strictly required with high precision. For this reason, $K_{0}$ could better be derived by the following relationship:

$$
K_{0}=\sqrt{2 B_{0} \frac{\varepsilon}{\tau}}
$$

rigorously valid for non-interconnected pores [5], but already successfuly employed in [9] for microporous filters.

\subsubsection{Isobaric Transport-controlled Conversion Runs}

When the fixed bed is operated with separate feed of reactants in the absence of a pressure difference and an instantaneous reaction takes place in the membrane, reactant concentration profiles as shown in Figure $3 \mathrm{c}$ can be expected. Since there is no pressure difference over the membrane and the pores are relatively large, the conversion rate is completely dominated by molecular diffusion. According to a simplified approach, the mass transfer in the porous medium can be described through Fick's law for both reactants as follows:

$$
\begin{gathered}
N_{a}=D_{a}^{e f f} \frac{C_{a}^{i}}{\delta_{r}} \\
N_{b}=D_{b}^{e f f} \frac{C_{b}^{i}}{\left(\delta-\delta_{r}\right)} .
\end{gathered}
$$

If Eqs. (5) and (6) are combined and the stoichiometry of the reaction is taken into account, the following equation can be derived:

$$
N_{a}=\frac{D_{a} c_{a}\left\{1+\left(D_{b} c_{b} / \nu_{b} D_{a} c_{a}\right)\right\}}{\delta} ; \quad\left(\nu_{a}=1\right)
$$


Due to the relatively large thickness of the barrier the external mass transfer resistance can again be neglected, thereby obtaining:

$$
R T \delta \frac{N_{a}}{p_{a}}=D_{a}^{e f f}+\frac{D_{b}^{e f f}}{\nu_{b}} \frac{p_{b}}{p_{a}}
$$

As a consequence, by varying the ratio of the partial pressures of the reactants at opposite barrier sides $\left(p_{a}, p_{b}\right)$ and measuring the converted flux of the key reactant under transport controlled conditions $\left(N_{a}\right)$, the effective diffusion coefficients of both reactants can be estimated. The $\varepsilon / \tau$-ratio can then be estimated from these effective diffusion coefficients and the calculated bulk diffusion ones according to Eq. (2). The bulk diffusion coefficients of the reactants can be estimated with acceptable approximation by the above-mentioned Fuller method, as if they were diffusing into pure nitrogen.

\subsubsection{Conversion in the Presence of a Pressure Difference}

Once the $\varepsilon / \tau, B_{0}$ and $K_{0}$ of both the filters and the fixed bed have been cstimated, the model can be validated for the situation of chemical reaction in the barrier accompanied with a pressure difference over the membrane, under complete mass transfer control (infinitely fast kinetics). In order to achieve such conditions, a well known, simple and very fast reaction was selected: the catalytic oxidation of carbon monoxide over supported platinum

$$
\mathrm{CO}+\frac{1}{2} \mathrm{O}_{2} \stackrel{\mathrm{Pt}}{\longrightarrow} \mathrm{CO}_{2}
$$

This reaction can be regarded to proceed instantaneously compared to the mass transfer rates, provided the operating temperature is high enough (see e.g. [10]), as later on checked (Section 4).

\section{THE EXPERIMENTAL SET-UP}

A schematic representation of the experimental set-up is shown in Figure 4. Inlet gas mixtures of either carbon monoxide or air depending on the barrier side are introduced into the reactor via mass flow controllers (Brooks). 


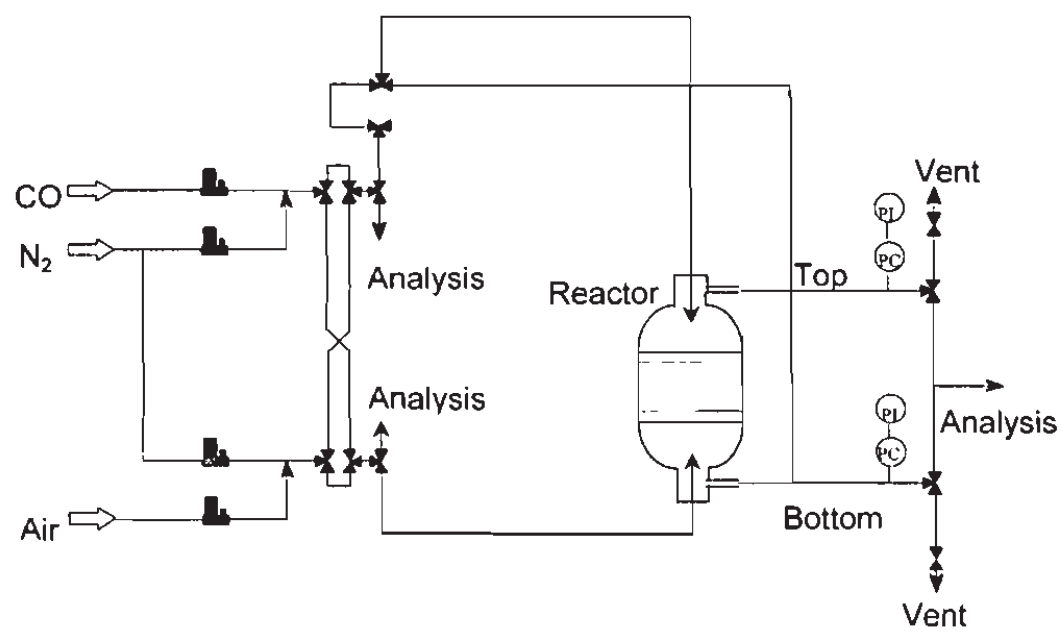

FIGURE 4 Experimental set-up.

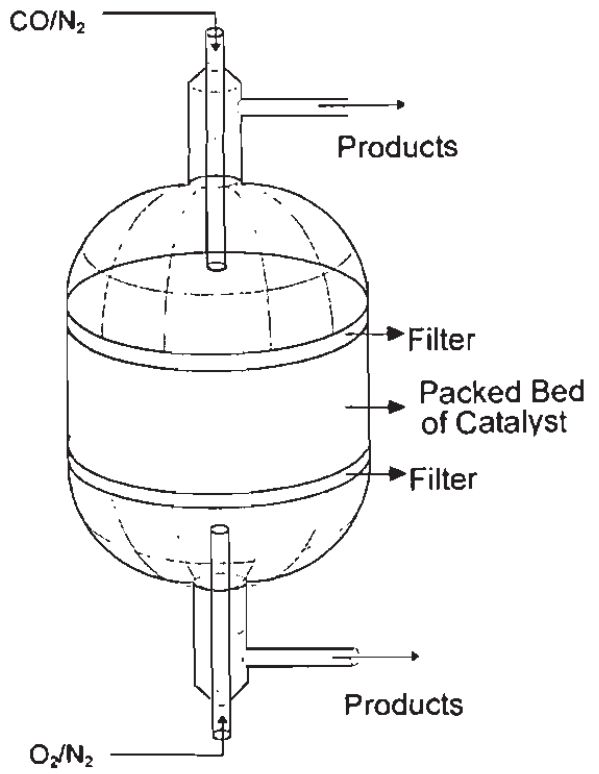

FIGURE 5 Sketch of the fixed bed barrier reactor (FBBR).

Nitrogen can also be dosed at both sides in order to change reactants concentration when required. Each gas mixture can either be fed to the top or the bottom of the reactor, or be analysed directly (reactor by-pass). 
The reactor is quartz-made and consists of an ordinary packed bed of a Pt-catalyst supported on lanthanum-stabilised transition alumina $\left(5<d_{n}<50 \mu \mathrm{m}\right)$, which is sandwiched between two porous silica filters (Fig. 5). The packed bed has a diameter of $45 \mathrm{~mm}$ and a thickness of $38 \mathrm{~mm}$. The two filters both have a thickness of $7 \mathrm{~mm}$ and an average pore size of $105 \mu \mathrm{m}$, as determined by $\mathrm{Hg}$ porosimetry. The reactor is surrounded by an electrical oven and temperatures are measured via $K$-type thermocouples in the heart of the packed bed and at the external surface of the reactor module. The pressure in both chambers is controlled by back pressure regulators and monitored by digital pressure transducers (Druck).

The gas streams can be analysed with $\mathrm{CO}$ (Maihak Defor) and $\mathrm{CO}_{2}$ (Maihak Unor 610) analysers. The volumetric flow rates leaving the reactor or by-passing it are determined with a bubble flow meter.

\section{RESULTS AND DISCUSSION}

\subsection{Diffusion Experiments (Determination of $\varepsilon / \tau$ )}

The diffusion experiments were carried out by feeding the bottom side of the membrane with a CO containing nitrogen mixture $\left(x_{\mathrm{CO}}=0.016-0.045\right)$ while the top side was fed with pure nitrogen. The diffusion experiments were carried out at room temperature $(T=293 \mathrm{~K})$ and atmospheric pressure. By measuring the fraction of $\mathrm{CO}$ at the outlet of the top and the bottom sides, both the diffusive flux and the driving force can be estimated on the basis of simple mass balances. The $\varepsilon / \tau$ ratio (derived through Eq. (2)) is plotted in Figure 6 as a function of the applied $\mathrm{CO}$ partial pressure difference. From this figure an average $\varepsilon / \tau$ value of about 0.32 can be estimated.

\subsection{Permeation Measurements (Determination of $B_{0}$ )}

The permeability measurements were carried out at room temperature $(T=293 \mathrm{~K})$ and average pressures varying from $1-3 \mathrm{bar}$. A flow of nitrogen or hydrogen $(320 \mathrm{Nml} / \mathrm{min})$ was fed to the top side of the barrier and the pressure difference over the barrier was measured. The obtained results are shown in Figure 7. It can be seen that the value for the permeability coefficient is measured accurately, because for both nitrogen and hydrogen nearly the same value of the permeability coefficient $\left(B_{0}=\sim 2.1 \cdot 10^{-13}\right)$ has been obtained experimentally. Analogous permeation runs performed on a 


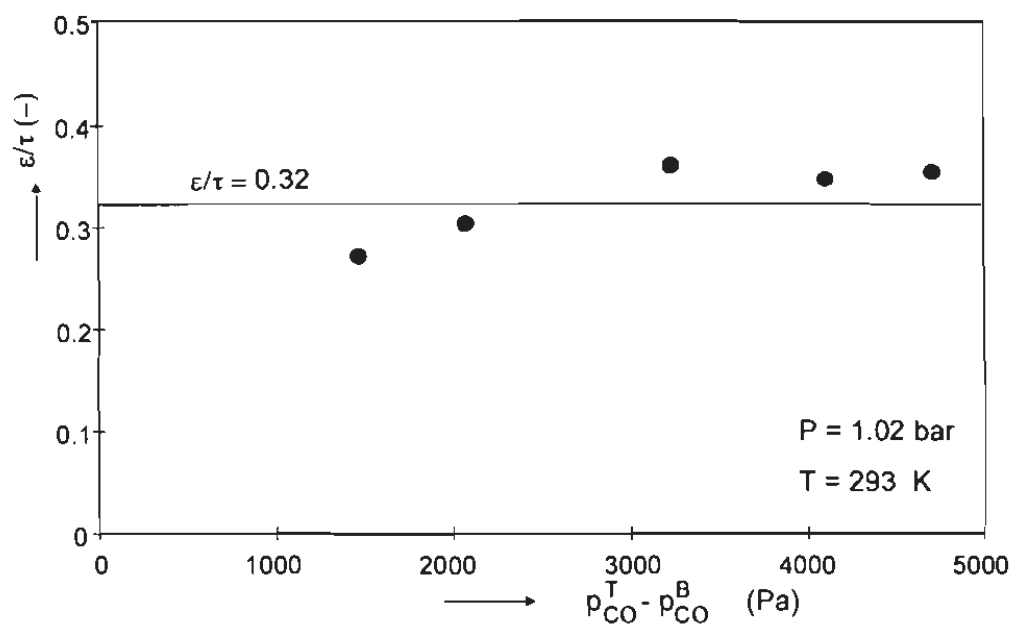

FIGURE 6 Results of diffusion experiments.

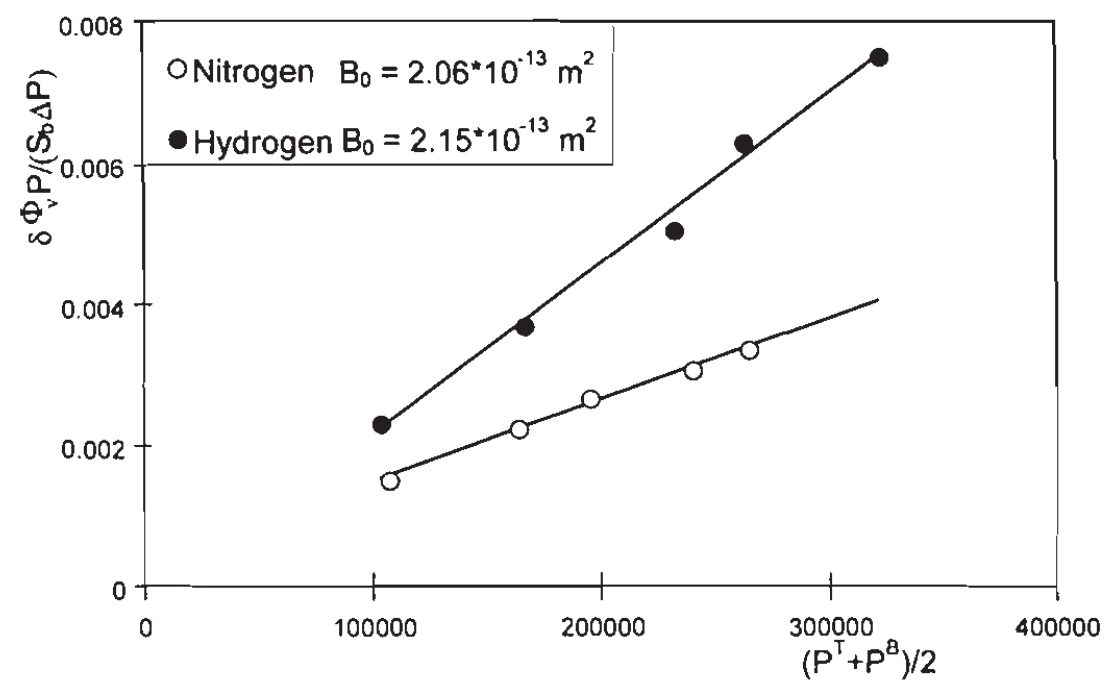

FIGURE 7 Results of permeation experiments.

single porous silica filter allowed to estimate a permeability $B_{0}^{\text {r }}$ of $3.6 \cdot 10^{-10} \mathrm{~m}^{2}$ for this porous media. On the grounds of Eq. (4) and on the mean value of $\varepsilon / \tau$ of 0.29 (see next section) the Knudsen factors of the fixed bed $\left(K_{0}=3.49 \cdot 10^{-6} \mathrm{~m}\right)$ and the filters $K_{0}^{\mathrm{f}}=1.44 \cdot 10^{-3} \mathrm{~m}$ can be easily estimated. 


\subsection{Isobaric Transport-controlled Conversion Experiments (Estimation of $\varepsilon / \tau$ )}

A different way to determine the $\varepsilon / \tau$-ratio is that of performing an instantaneous reaction in the barrier under isobaric conditions. As above mentioned, a preliminary issue concerning the present type of characterisation is the need to operate in a transport controlled regime. This was easily checked by studying the influence of temperature on the overall $\mathrm{CO}$ conversion at given operating conditions. A carbon monoxide/nitrogen mixture $\left(x_{\mathrm{CO}}=0.048\right)$ is fed to the bottom chamber of the reactor and an oxygen/nitrogen mixture $\left(x_{\mathrm{O}_{2}}=0.043\right)$ was fed to the top one. The experiments were carried out at 1.5 bar in the absence of a pressure diffcrence over the FBBR reactor and at a temperature ranging from 516 to $756 \mathrm{~K}$. The results from this conversion experiment are plotted in Figure 8 as an Arrhenius plot. From the slope of Figure 8 an apparent activation energy of $3 \mathrm{~kJ} / \mathrm{mol}$ was found, a low enough value to indicate that the conversion rate is indeed completely mass transfer limited over the entire temperature range tested.

In Section 2.3.3 it was shown that, under transport controlled conditions and in the absence of absolute pressure difference between the two sides of the fixed bed, by varying the ratio of partial pressures of the reactants on both sides the effective diffusion coefficient of both key reactants could be estimated (see Eq. (8)). An oxygen/nitrogen mixture $\left(x_{\mathrm{O}_{2}}=0.043\right)$ was fed

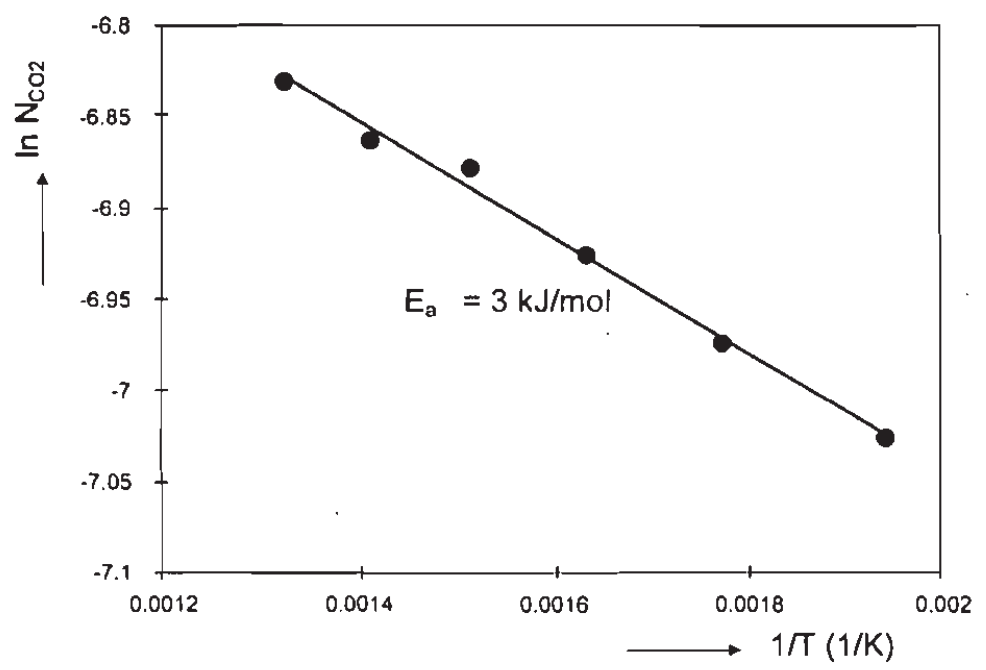

FIGURE 8 Arrhenius plot for the catalytic oxidation of $\mathrm{CO}$ in a FBBR. 
to the top, while a carbon monoxide/nitrogen mixture $\left(x_{\mathrm{CO}}=0.015-0.050\right)$ was fed to the bottom. The experiment was carried out in the absence of a pressure difference over the barrier ( 1.5 bar at both sides) and at $753 \mathrm{~K}$ (conservatively fixed at the highest values of the temperature range tested earlier). The results obtained are shown in Figure 9, where the left-hand-side of Eq. (8) is plotted against the ratio between the partial pressure of oxygen at the top and the partial pressure of carbon monoxide at the bottom of the reactor. The effective diffusion coefficient of oxygen and carbon monoxide can be estimated from the slope and intercept of this line, respectively (see Eq. (3)). The obtained values resulting from these experiments are presented in Table II. It can be seen that the quantitative value of the observed $\varepsilon / \tau$ ratio obtained from both effective diffusion coefficients do not perfectly overlap. This deviation is possibly caused by the use of the Fuller-equation at elevated temperature. However, an average $\varepsilon / \tau$ value of 0.26 can be estimated. In the following, the mean value 0.29 between the diffusion-based and the isobaric-conversion-based estimates will be used for modelling purposes. This value is in good agreement with theoretical value for random

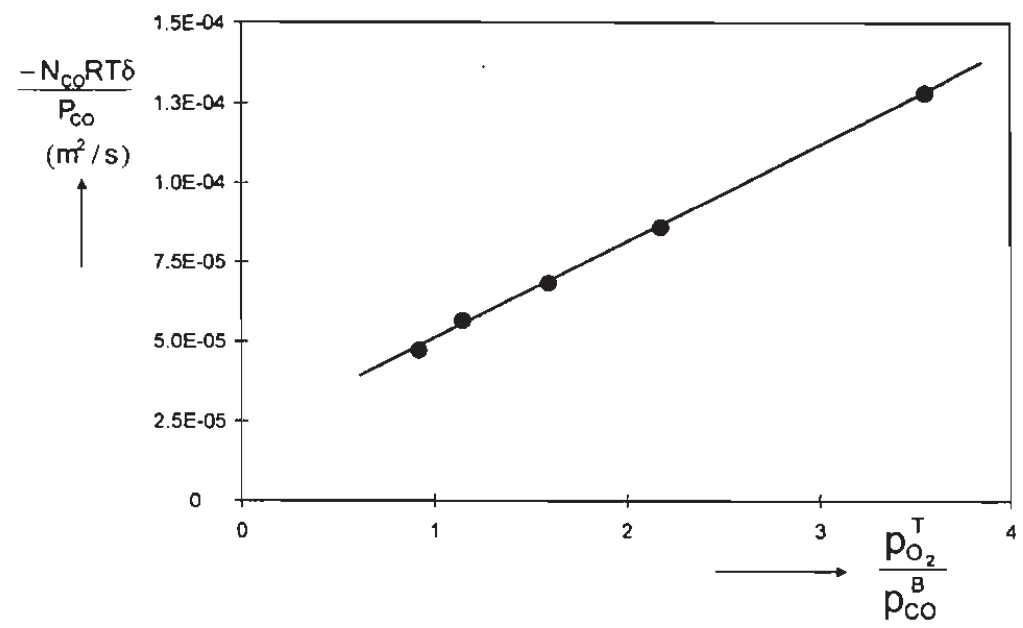

FIGURE 9 Results of isobaric conversion experiments.

TABLE II Results of isobaric conversion experiments

\begin{tabular}{lccc}
\hline & $\begin{array}{c}D^{e I J} \\
\left(\mathrm{~m}^{2} / \mathrm{s}\right)\end{array}$ & $\begin{array}{c}D^{0}(\text { Fuller }) \\
\left(\mathrm{m}^{2} / \mathrm{s}\right)\end{array}$ & $\begin{array}{c}\varepsilon / \tau \text {-ratio } \\
(-)\end{array}$ \\
\hline Carbon monoxide & $2.1 \cdot 10^{-5}$ & $7.1 \cdot 10^{-5}$ & 0.29 \\
Oxygen & $1.6 \cdot 10^{-5}$ & $7.1 \cdot 10^{-5}$ & 0.23 \\
\hline
\end{tabular}


ordered packed beds [11] which is given as:

$$
\tau=\frac{1}{\sqrt{\varepsilon}}
$$

or

$$
\frac{\varepsilon}{\tau}=\varepsilon \sqrt{\varepsilon} .
$$

This approach gives an $\varepsilon / \tau$-ratio of 0.28 as for a dense packed bed when $\varepsilon$ is assumed equal to 0.43 (spherical particles).

\subsection{Conversion Experiments in the Presence of a Pressure Difference Over the Barrier}

In these final investigations an oxygen/nitrogen mixture $\left(x_{\mathrm{O}_{2}}=0.043\right)$ was fed to the top, while a carbon monoxide/nitrogen mixture $\left(x_{\mathrm{CO}}=0.048\right)$ was fed to the bottom of the reactor. The experiments were carried out at a pressure of 1.5 bar at the bottom side, while the pressure difference over the membrane (high pressure on the top side) was increased from $0-100 \mathrm{mbar}$. The $\mathrm{CO}_{2}$ production was measured at both sides and plotted as a function of the pressure difference in Figure 10. The applied pressure difference over the barrier has both an influence on the distribution of the products and also on the total production rate of $\mathrm{CO}_{2}$. An increase in the pressure difference will

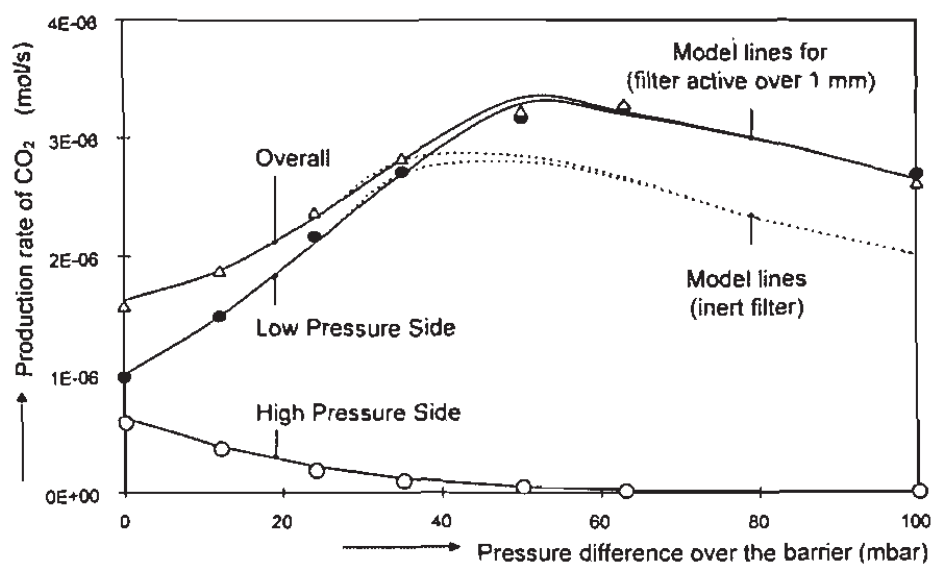

FIGURE 10 Conversion rate of $\mathrm{CO}$ to $\mathrm{CO}_{2}$ as a function of the pressure difference over the mcmbrane (experimental data and model lines). 
preferably direct the product to the low pressure side of the reactor. This behaviour is illustrated in Figure 10, where the $\mathrm{CO}_{2}$ directed to the low pressure side is initially increasing, while that of the high pressure side is decreasing. At pressure differences higher than $50 \mathrm{mbar}$, the generated $\mathrm{CO}_{2}$ is almost completely pushed to the low pressure side. It can also be seen that the applied pressure difference initially has a positive effect on the total conversion rate, which reaches a maximum at approximately $50 \mathrm{mbar}$, meanwhile the reaction zone gradually shifts towards the low pressure side of the barrier. A further increase of the pressure difference results in a decrease of overall $\mathrm{CO}_{2}$ production rate, as a consequence of the absence of catalytic activity of the filter, reached by a more and more significant fraction of the reactant fed at the high-pressure chamber and remained unconverted.

\subsection{Model Calculations Versus Experimental Data}

The conversion experiments in the presence of a pressure difference cannot anymore be predicted by simple analytical equations as for the case of transport-controlled isobaric conversion (Eqs. (5-8)). The complete set of equations listed in Figure 1 has to be used. When attributing no catalytic activity to the filters, the dashed model lines in Figure 10 were the output of model calculations. In this case, the calculated conversion are, at first, quite in good agreement with the experimental data (low-to-medium pressure differences), but deviate from them quite significantly at medium-to-high $\Delta P$ values. The reason for this discrepancy lies in the assumption that the filters are completely inert. Microscopic inspection of the filters after the experimental work, allowed to detect catalytic particles penetrated into the filter matrix, making the filters partially active, which has also been reported by Neomagus et al. [12] for the partial oxidation of isobutene. When the filters are assumed to be active over a distance of just $1 \mathrm{~mm}$ close to the fixed bed the continuous model lines in Figure 10 are obtained, showing a much better agreement with the experimental values at medium-to-high pressure differences. This catalyst penetration thickness is essentially a fitting parameter, since it is difficult to estimate experimentally. Its value is though quite reasonable since the total thickness of the filter is $7 \mathrm{~mm}$.

As far as the achievable $\mathrm{CO}$ conversion is concerned, a maximum of $20.5 \%$ was calculated for a space velocity of about $30 \mathrm{~min}^{-1}$, referred to the fixed-bed volume. This figures could likely be increased in an industrial application perspective by e.g., reducing the thickness of the filters and of the fixed bed. However, the major goal of the FBBR is not to substitute at 
an industrial level membrane reactors, but to provide a lab-scale alternative, easier to handle, to these counterparts.

In Figure 11, the mole fraction profiles of the product and the reactants are given in the absence of pressure difference (Fig. 11a) and for an applied pressure difference of $50 \mathrm{mbar}$ (Fig. 11b). From these figures, it can be clearly seen that, for $\Delta P=0$ mbar, the reaction zone is located a bit closer to
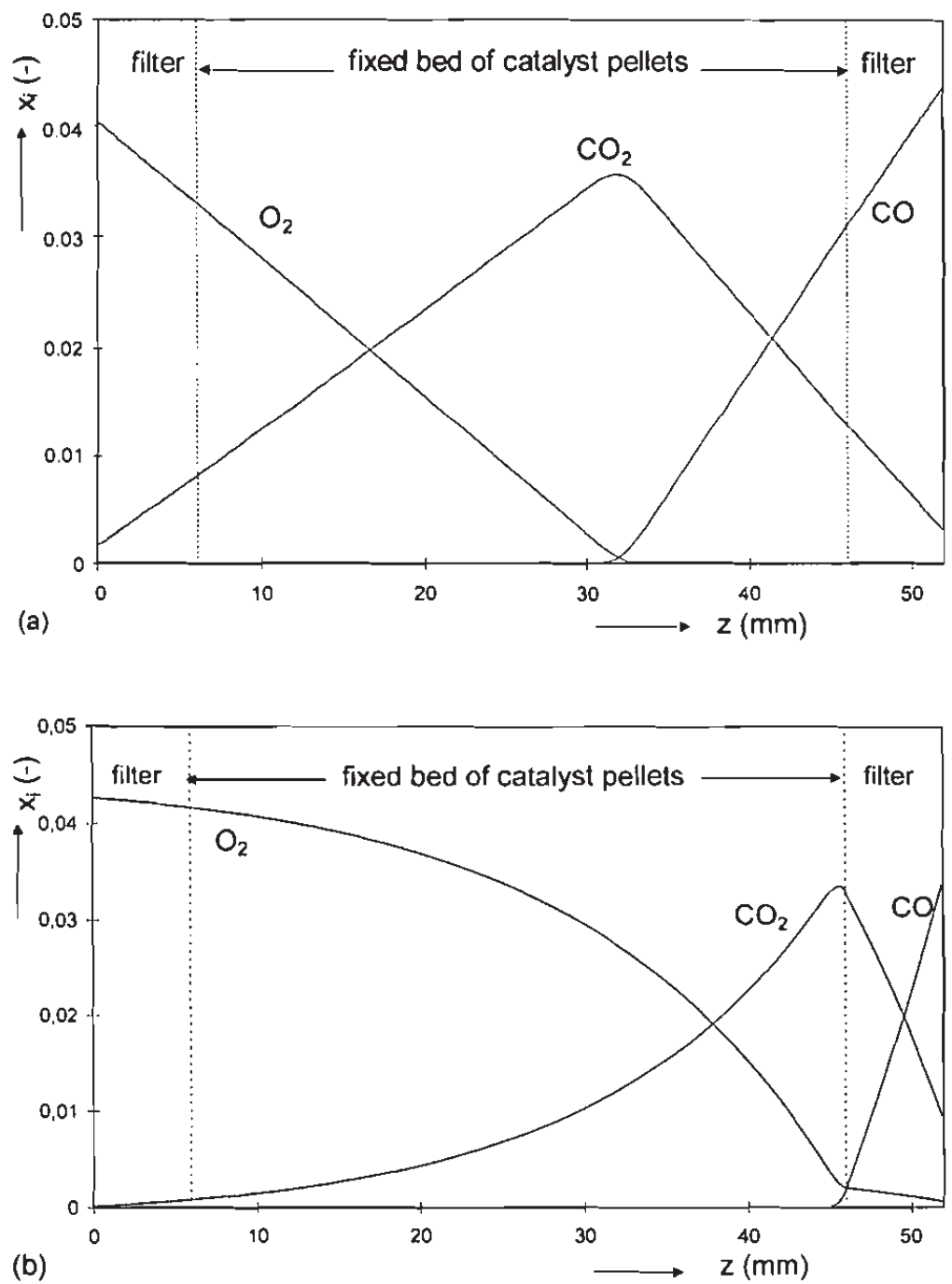

FIGURE 11 Calculated mole fraction profiles in the absence of a pressure difference (a) and with a pressure difference of $50 \mathrm{mbar}$ (b). 
the $\mathrm{CO}$-side, and that, at a pressure difference of $50 \mathrm{mbar}$, the reaction zone is actually shifted towards the inactive layer of the barrier (low-pressure-side filter). Normally, if reaction kinetics are high enough, an increase of the pressure difference increases the overall conversion rate, since it will generate a convective flow counterbalanced by a up-wind diffusion of the reactant fed at the low pressure side (negative effect), which also increases due to the shift of the reaction zone that reduces the diffusion path [4]. This conditions do not hold any longer when the reaction plane is pushed outside the catalyst location. Since the filters are not active (at least beyond a given distance from the fixed bed), the conversion rate obviously decreases as the reaction zone has reached the catalytically-inert zone.

\section{CONCLUSIONS}

A fixed bed barrier reactor with separate feed of reactants has been conceived and tested. The reactor consists of a fixed bed of small catalytic particles kept between two filters. The reactants are not premixed, but are fed separately to the reactor at the two sides of the barrier. Due to this configuration an active and homogeneous catalytic barrier is obtained as an alternative to formerly studied non-permselective membrane reactors [2$4,6,12]$ based on thin catalytic membranes having pores in the KnudsenPoiseuille transition range.

The performance of the fixed-bed barrier reactor was characterised using the catalytic oxidation of carbon monoxide as a test reaction, after evaluating the most important structural parameters of the barrier, $B_{0}, K_{0}$ and $\varepsilon / \tau$, by permeation, diffusion and isobaric conversion experiments. Using this structural parameters in a reactor model, based on the Dusty Gas Model [5], conversion rates in the presence of a pressure difference could be predicted accurately, with the only exception of high pressure drop conditions, where a fitting parameter (thickness of the catalyst powder penetration in the sandwiching filters) had to be used.

As a consequence, the fixed-bed barrier reactor can be regarded as a good alternative to the non-permselective membrane reactor, at least at a lab scale, for carrying out reactions according to a separate-feed of reactants concept. However, a deeper comparison between these two reactor concepts is attempted in Table III, trying to assess how they fit different evaluation criteria.

As a main conclusion it can be stated that the membrane reactor offers some technological advantages over the fixed-bed barrier reactor: lower 


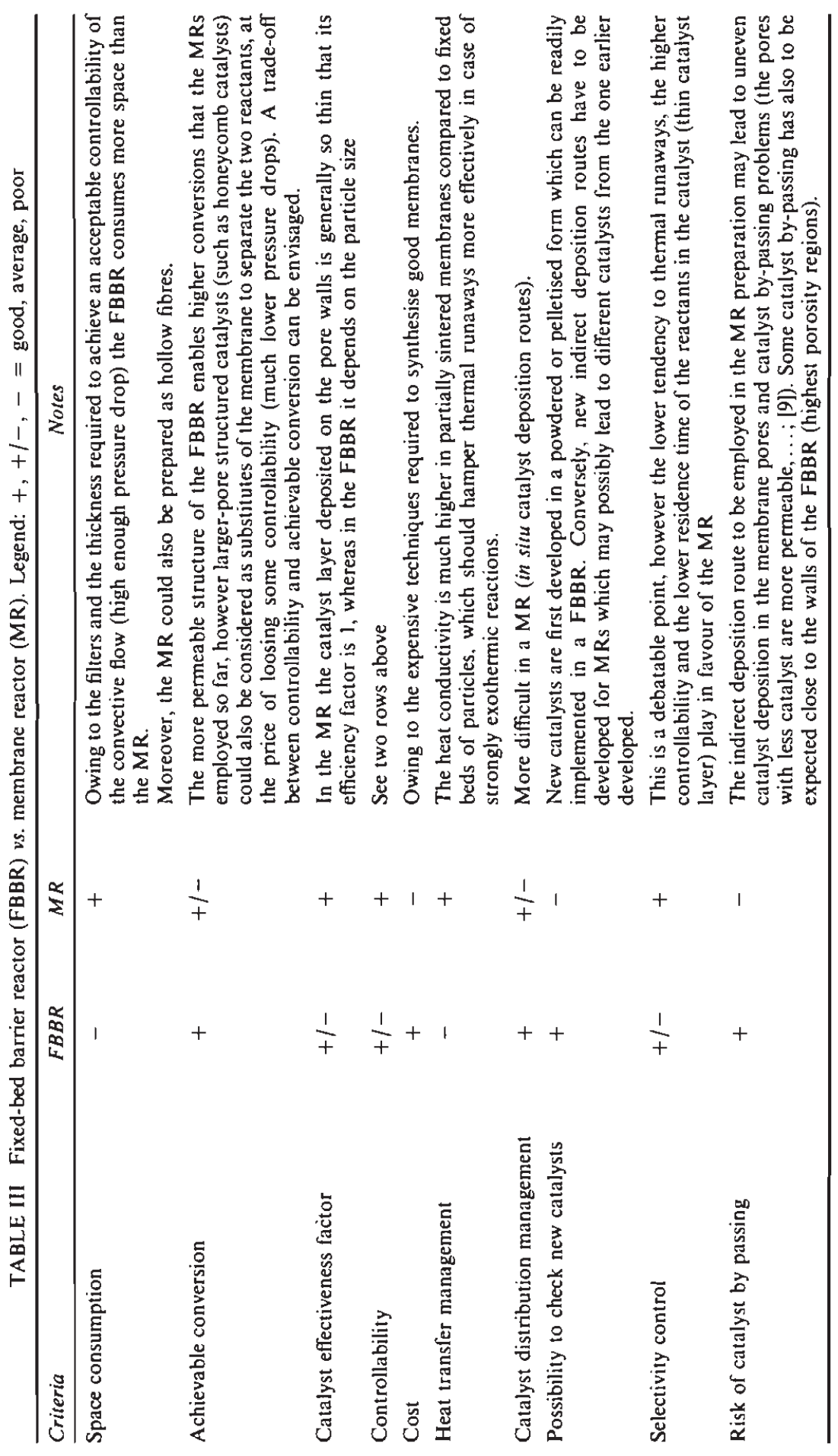


space consumption (hollow fibres), better heat management (higher thermal conductivity owing to its partially-sintered nature), high effectiveness factor (the catalyst layer deposited on the pore walls is very thin), ... However, the membrane reactor needs high-tech preparation processes (hollow fibres technology; indirect catalyst deposition routes capable of avoiding pore plugging as well as catalyst bypassing, and allowing a desired catalytic activity distributions; sealing of membranes into modules; etc.) to fully exploit this potential. It has though to be underlined that such technological challenges are certainly lower than those to be faced in the attempt to synthesise highly-permselective membranes [13]. Until the mentioned membrane preparation routes will be successfully available at a convenient scale and price, the fixed-bed barrier reactor will have to be considered as the preferential tool to check the potential of the separate feed of reactants concept in new applications and to expand it, if appropriate, to an industrial level. At a lab scale, issues like space consumption are indeed less important and the possibility to check readily new catalyst formulations with a reliable activity distribution throughout the reactor is highly welcome.

\section{Acknowledgements}

The authors want to acknowledge GASTEC NV (Apeldoorn, The Netherlands) for their financial support, B. T. J. Oosterlee for his experimental work and $\mathrm{H}$. J. Moed for his technical assistance.

\section{NOTATION}

$B_{0} \quad$ permeation coefficient $\left(\mathrm{m}^{2}\right)$

$c$ concentration $\left(\mathrm{mol} \mathrm{m}^{-3}\right)$

$d_{p}$ particle diameter $(\mathrm{m})$

$D$ diffusion coefficient $\left(\mathrm{m}^{2} \mathrm{~s}^{-1}\right)$

$E_{\mathrm{a}} \quad$ activation energy $\left(\mathrm{kJ} \mathrm{mol}^{-1}\right)$

$K_{0} \quad$ Knudsen coefficient $(\mathrm{m})$

$M$ molar mass $\left(\mathrm{kg} \mathrm{mol}^{-1}\right)$

$N$ molar flux ( $\left.\mathrm{mol} \mathrm{m}^{-2} \mathrm{~s}^{-1}\right)$

$p \quad$ partial pressure $(\mathrm{Pa})$

$P$ pressure $(\mathrm{Pa})$

$Q \quad$ production/conversion rate $\left(\mathrm{mol} \mathrm{s}^{-1}\right)$

$R \quad$ ideal gas constant $=8.314 \mathrm{~J} \cdot \mathrm{mol}^{-1} \cdot \mathrm{K}^{-1}$ 
$\mathfrak{R}$ reaction rate $\left(\mathrm{mol} \mathrm{m}^{-3} \mathrm{~s}^{-1}\right)$

$T$ temperature (K)

$S_{b} \quad$ cross-sectional area of the barrier $\left(\mathrm{m}^{2}\right)$

$x$ mole fraction

$z \quad$ axial co-ordinate through the barrier (m)

\section{Greek Symbols}

$\delta \quad$ thickness of the barrier, including fixed bed and filters ( $\mathrm{m}$ )

$\varepsilon \quad$ porosity

$\mu \quad$ viscosity (Pa s)

$\nu \quad$ stoichiometric coefficient

$\phi_{v} \quad$ volumetric flow $\left(\mathrm{m}^{3} \mathrm{~s}^{-1}\right)$

$\tau$ tortuosity

\section{Subscripts}

$a, b$ reactants

$i \quad$ component $i$

$j \quad$ component $j$

$K \quad$ Knudsen

$p \quad$ products

\section{Superscripts}

$\begin{array}{ll}\text { B } & \text { bottom } \\ \text { ef } & \text { effective } \\ \text { f } & \text { filter } \\ \text { H } & \text { high } \\ \text { i } & \text { interface } \\ \text { L } & \text { low } \\ \text { T } & \text { top } \\ 0 & \text { bulk }\end{array}$

\section{References}

[1] Sloot, H. J., Versteeg, G. F. and van Swaaij, W. P. M. (1990). Chem. Engng. Sci., 45, 2415.

[2] Veldsink, J. W., van Damme, R. M. J., Versteeg, G. F. and van Swaaij, W. P. M. (1992). Chem. Engng. Sci., 47, 2939.

[3] Saracco, G. and Specchia, V. (2000). Catalytic combustion of propane in a membrane reactor with separate feed of reactants IV. Transition from the kinetics- to the transportcontrolled regime, Chem. Eng. Sci., 55, 3979-3989. 
[4] Neomagus, H. W. J. P., van Swaaij, W. P. M. and Versteeg, G. F. (1998). J. Membr. Sci., $148,147$.

[5] Mason, E. A. and Malinauskas, A. P., Gas transport in porous media: The dusty gas model (Elsevier, Amsterdam, 1983).

[6] Saracco, G., Veldsink, J. W., Versteeg, G. F. and van Swaaij, W. P. M. (1995). Chem. Eng. Sci., 50, 2833.

[7] Fuller, E. N., Schettler, P. D. and Giddings, J. C. (1966). Ind. Eng. Chem. Res., 58, 18.

[8] Bird, R. B., Stewart, W. E. and Lightfoot, E. N., Transport Phenomena (Wiley, New York, 1960).

[9] Saracco, G. and Specchia, V. (1995). Ind. Eng. Chem. Res., 34, 1480.

[10] Venderbosch, R. H., Prins, W. and van Swaaij, W. P. M. (1998). Chem. Engng. Sci., 53, 3355.

[11] Punchochar, M. and Drahos, J. (1993). Chem. Eng. Prog., 48, 141.

[12] Neomagus, H. W. J. P., van Swaaij, W. P. M. and Versteeg, G. F. (1999). Chapter 5 in H. W. J. P. Neomagus' Ph.D. Thesis, University of Twente, Enschede, The Netherlands.

[13] Saracco, G., Neomagus, H. W. J. P., Versteeg, G. F. and van Swaaij, W. P. M. (1999). Chem. Eng. Sci., 53, 1997. 